\title{
An appointment lead time of more than two weeks is significantly associated with not presenting for a liver biopsy procedure*
}

\author{
Hasan Yilmaz \\ Division of Gastroenterology, Department of Internal Medicine, Kocaeli University School of Medicine, Kocaeli, Turkey
}

\begin{abstract}
Background and Aim: A liver biopsy is the gold standard diagnostic tool for liver disease. Patient failure to attend a liver biopsy appointment causes a delay in diagnosis and the initiation of therapy. The aim of this study was to determine factors associated with liver biopsy appointment noncompliance.

Materials and Methods: A cross-sectional study was used to analyze the data of consecutive outpatients with liver disease who had a liver biopsy appointment at a tertiary university hospital hepatology center between March 2020 and March 2021. Baseline demographic information and logistical factors that might affect presentation for an appointment were examined.

Results: A total of 82 patients ( $50 \%$ female) with a mean age of $45 \pm 12.31$ years were included in the study. The rate of not attending the biopsy appointment was $15.9 \%$. Age, sex, distance to the healthcare facility, and patient disease were not linked to appointment adherence, however, attendance was significantly associated with an appointment date made more than 12 days in advance, with $85 \%$ sensitivity and $72 \%$ specificity (area under the curve: $0.809 ; 95 \%$ confidence interval: $0.708-0.888$; $\mathrm{p}<0.0001$ ).
\end{abstract}

Conclusion: The nonattendance rate for liver biopsy appointments is relatively high, and it is an obstacle to the effectiveness of hepatology clinics. Making appointments for patients within 2 weeks may increase liver biopsy attendance.

Keywords: Appointment adherence; lead time; liver biopsy.

\section{Introduction}

Percutaneous ultrasound (USG)-guided liver biopsy (PLB) is one of the most widely used methods to identify liver pathologies. The method is quite simple, the training period is short, and the complication rates are low, compared with many interventional biopsy procedures. Recent research has suggested that the major complication rate is $0.57 \%$ to *The results of this study were presented as an oral presentation at the $17^{\text {th }}$ National
Hepato-Gastroenterology Congress held on June $17-19,2021$.

How to cite this article: Yilmaz $\mathrm{H}$. An appointment lead time of more than two weeks is significantly associated with not presenting for a liver biopsy procedure. Hepatology Forum 2021; 2(3):107-111.

Received: August 24, 2021; Accepted: September 08, 2021; Available online: September 00, 2021

Corresponding author: Hasan Yilmaz; Kocaeli Universitesi Tip Fakultesi, Ic Hastaliklari Anabilim Dali, Gastroenteroloji Anabilim Dali, Istanbul, Turkey Phone: +90 50587748 21; e-mail: hasan.yilmaz@kocaeli.edu.tr (c) (i) (8) OPEN ACCESS

This work is licensed under a Cretive Commons Attribution-NonCommercit 40 interational License C Copyright 2021 by Hepatology Forum - Available online at www.hepatologyforum.org
$3.1 \%,{ }^{[1,2]}$ and mortality related to the procedure has been reported to be $0.1 \%$ to $0.3 \%{ }^{[1,3]}$ It is broadly agreed that a liver biopsy is a safe and effective procedure.

Due to various etiologies, a liver biopsy is most frequently used in the diagnosis, staging, and follow-up of viral hepatitis and nonalcoholic steatohepatitis (NASH) in daily practice. Although there are now non-invasive methods, such as the FibroTest (BioPredictive, Paris, France), and elastography, that can successfully determine the stage of liver fibrosis, a liver biopsy continues to be the mainstay for the diagnosis of liver disease. Though the area under the receiver operating curve (AUROC) of the FibroTest is between 0.70 and 0.80 , suggesting that it is not a perfect or superior diagnostic tool when compared with PLB, it appears to be a reasonable option. ${ }^{[4]}$ However, a significant inter-laboratory variation has been noted with the FibroTest that can cause a misdiagnosis of significant fibrosis in as many as $20 \%$ of patients. ${ }^{[5]}$ FibroScan (Echosens, Paris, France) is another pain-free method that can be used quickly and easily. However, Ziol et al. ${ }^{[6]}$ determined an AUROC value of 0.79 for $\mathrm{F} \leq 2,0.91$ for $\mathrm{F} \leq 3$, and 0.97 for $\mathrm{F}=4$. The authors concluded that this test has good value for minimal and significant fibrosis, but was limited for intermediate fibrosis. It is also important to note that the cost of a FibroScan device (about 80,000 euros at the time of writing) is high for most developing countries. As a result, the utility of a liver biopsy is increasing, despite the availability of noninvasive techniques. ${ }^{[7]}$ Furthermore, liver biopsy indications include, but are not limited to, diffuse hepatopathies, toxic hepatitis, metabolic liver disease (Wilson's Disease, hemochromatosis), infiltrative pathologies (Gaucher, amyloidosis), primary biliary cholangiopathy, primary sclerosing cholangitis, autoimmune hepatitis, and liver transplant rejection. Thus, a liver biopsy remains the gold standard test for parenchymal pathologies of the liver.

Patient failure to present at scheduled appointments causes delays in diagnosis and treatment. It also leads to an inefficient use of healthcare resources. When patients do not attend appointments, treatment is delayed, the number of patients on waiting lists increases, and accordingly, patient satisfaction decreases. In other words, determining the reasons for nonattendance at liver biopsy appointments and developing effective solutions will enable hepatology clinics to function more effectively. Although the rate of appointment noncompliance varies in different fields of healthcare, and the factors that may influence this failure to appear have been widely studied in recent years, to the best of our knowledge, there are no published data related specifically to liver biopsy appointment nonattendance.

This study was designed to determine the nonattendance rate for a liver biopsy to better understand the impact on healthcare. Factors that may influence appointment compliance behavior were targeted in order to develop measures that could improve the effectiveness of hepatology clinics. 


\section{Materials and Methods}

\section{Study Design and Data Source}

Data of patient nonattendance at an appointment for a liver biopsy at a tertiary gastroenterology and hepatology center between March 2020 and March 2021 were retrospectively assessed. Details of patient age, gender, liver biopsy indication, marital status, season of appointment, travel distance, and travel time between the home address of the patient and the hospital, malignancy as a comorbidity, and the time between making the appointment and the date of the appointment (lead time) were extracted from hospital's electronic data system for analysis as independent variables related to failure to keep a liver biopsy appointment. The dependent variable was failure to attend a scheduled biopsy appointment.

All consecutive patients with liver disease who were $>18$ years of age and had an appointment for a liver biopsy during the study period were included. The patients excluded from the study were those with liver biopsies performed by an interventional radiologist, those with inconsistent data in the electronic records, those whose appointment was canceled by the physician or institution, appointments scheduled for hospitalized patients, and appointments canceled by patients 48 hours before the appointment date or more.

\section{Ethical Considerations}

This study was performed according to the Helsinki Declaration. Written, informed consent was obtained from all of the patients before liver biopsy. This study was a subanalysis of a previous retrospective analysis of absenteeism rates and relevant factors at endoscopy units, conducted with the approval of the Kocaeli University Faculty of Medicine Non-Invasive Clinical Research Ethics Committee on February 18, 2021 (identifier: GOKAEK-2021/4.26, project no: 2021/79). The results of this study were presented as an oral presentation at the $17^{\text {th }}$ National Hepato-Gastroenterology Congress held online June 17-19, 2021.

\section{Liver Biopsy Procedure}

All of the liver aspiration biopsies were performed with USG guidance using the Menghini technique. For each liver biopsy, it was ensured that the platelet count of the patient was greater than $>70000 \mu \mathrm{L}$, the partial thromboplastin time was $<50$ seconds, and that they were not receiving anticoagulant drugs. Premedication of $50 \mathrm{mg}$ tramadol and $1 \mathrm{mg}$ midazolam was provided before all of the procedures, and the biopsy localization and needle tract were determined using USG. A HEPAFIX Lok G 17 1.4-mm needle (B. Braun Melsungen AG, Melsungen, Germany) was used in all cases. A sandbag was positioned after the biopsy and the patients were observed for 4 hours. Blood pressure was checked hourly during the observation period. Before discharge, a complete blood count was ordered and a hemorrhage control was performed with USG, and patients who were discharged were followed up on an outpatient basis.

\section{Statistical Analysis}

All of the statistical analyses were performed using IBM SPSS Statistics for Windows, Version 20.0 (IBM Corp., Armonk, NY, USA) and MedCalc Statistical Software version 14 (MedCalc Software bv, Ostend, Belgium). Numeric variables were presented as the mean $\pm \mathrm{SD}$. Categorical variables were summarized using the count (percentage). Comparisons of numeric variables between groups were carried out us-

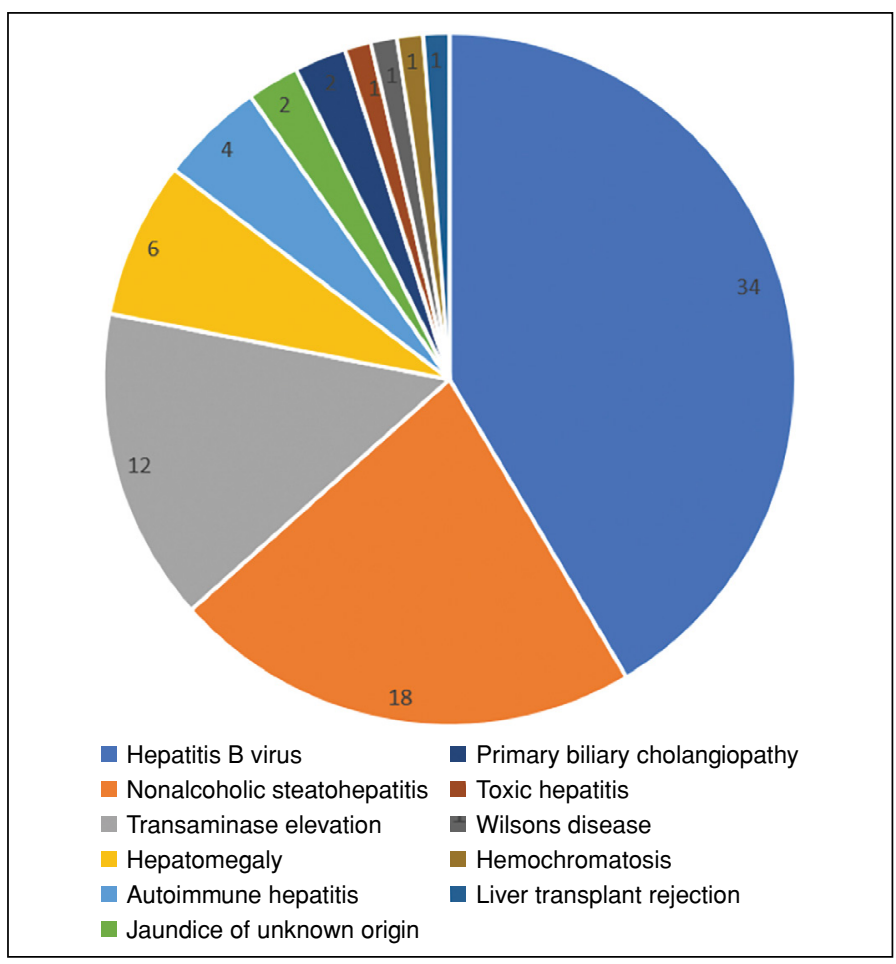

Figure 1. Liver biopsy indications.

ing an independent samples t-test/Mann-Whitney $U$ test, as appropriate. The association between 2 categorical variables was examined using a chi-squared test. Receiver operating characteristic (ROC) curves were used to analyze the capacity of lead time to detect nonattendance at an appointment. The Youden index was used to calculate the sensitivity and specificity. All of the statistical analyses have a 95\% significance level, and a 2 -sided $\mathrm{p}$ value of $<0.05$ was considered statistically significant.

\section{Results}

\section{Patient Characteristics}

Initially, 112 patients who did not present for a liver biopsy appointment were identified in the 1-year study period. Patients were excluded from the study as follows: 12 due to inconsistent data, 6 had an interventional radiologist perform the biopsy, 4 were inpatients, 4 were $<18$ years of age, and 5 had hemangioma in the needle tract detected with USG before the procedure. The final study group comprised 82 patients.

In all, 41 patients $(50 \%)$ were female. The mean age of the patients was $45.94 \pm 12.31$ years. While $74(90.2 \%)$ of the patients were $<65$ years of age and defined as a working-age group, $8(9.8 \%)$ were $\geq 65$ years of age. The majority of the patient population was of working age and were married $(\mathrm{n}=78[95.1 \%])$.

The most common biopsy indication was HBV virus infection. There was no significant difference between the reason for the biopsy and appointment compliance ( $\mathrm{p}=0.22$ ) (Fig. 1 ).

Seventy-three $(89 \%)$ patients traveled to outpatient clinics from within the inner city for the liver biopsy. The average travel distance between the home address of the patients and the biopsy center was $26.43 \mathrm{~km}$ (interquartile range [IQR]: 17.02-57.79), and the median travel time was calculated to be 32 minutes (IQR: 21.50-51.00). With regard to season and travel on the potential impact on attendance, $17(20.5 \%)$ 


\begin{tabular}{|c|c|c|c|}
\hline Variable & $\begin{array}{l}\text { Patients who } \\
\text { presented for } \\
\text { appointment } \\
(\mathrm{n} / \%)\end{array}$ & $\begin{array}{l}\text { Patients who } \\
\text { did not } \\
\text { present for } \\
\text { appointment } \\
(\mathrm{n} / \%)\end{array}$ & p \\
\hline Gender & & & 0.547 \\
\hline Female & $33(47.8)$ & $8(61.5)$ & \\
\hline Male & $36(52.2)$ & $5(38.5)$ & \\
\hline Age (years) & & & 0.606 \\
\hline$<65$ & 63 (91.3) & $11(84.6)$ & \\
\hline$>65$ & $6(8.7)$ & $2(15.4)$ & \\
\hline Marital status & & & 1.000 \\
\hline Married & $66(95.7)$ & $12(92.3)$ & \\
\hline Single & $3(4.3)$ & $1(7.7)$ & \\
\hline Referral & & & 0.613 \\
\hline Rural & $4(6.2)$ & $0(0.0)$ & \\
\hline Inner city resident & $61(93.8)$ & $12(100)$ & \\
\hline Season & & & 0.363 \\
\hline Spring & $15(21.7)$ & $2(15.4)$ & \\
\hline Summer & $19(27.5)$ & $3(23.1)$ & \\
\hline Fall & $20(29.0)$ & $7(53.8)$ & \\
\hline Winter & $15(21.7)$ & $1(7.7)$ & \\
\hline Comorbidity & & & 1.000 \\
\hline Malignant & $12(17.4)$ & $2(15.4)$ & \\
\hline Nonmalignant & $57(82.6)$ & $11(84.6)$ & \\
\hline
\end{tabular}

of the liver biopsies were performed in the spring, $22(26.8 \%)$ in the summer, 27 (32.9\%) in the autumn, and $16(19.5 \%)$ in the winter season. A comorbidity of nonhepatic malignancy was seen in $14(17.1 \%)$ of the patients (Table 1). The median length of time between making an appointment and the biopsy procedure was 8 days (IQR: 5.00-14.00).

\section{Comparison of Patients Who Did and Did Not Present for Biopsy}

The rate of nonattandance for a liver biopsy appointment was $15.9 \%$ (13/82). The interval between making a biopsy appointment and the procedure (lead time) was significantly longer among the patients who did not present for the appointment (Nonattender vs attender: 20 [IQR: 14-27] vs 8 [IQR: 5-14]; $\mathrm{p}=0.001)$ There was no significant difference between the nonattenders and attenders groups in terms of age (median: 51 years [IQR: 28-72] vs 44 years [IQR: 37-55]; $\mathrm{p}=0.240$ ), travel distance, [median: $17 \mathrm{~km}$ (IQR: 10-39) vs $26 \mathrm{~km}$ (IQR: 17-57 ; $\mathrm{p}=0.133$ ), and travel time (22 minutes [14-39] vs 32 minutes [IQR: $21-51] ; p=0.134)$. Examination of the working-age group, marital status, referral from within the city or outside the city, the season in which the biopsy appointment was scheduled, and nonhepatic malignant comorbidity variables revealed no significant difference (Table 1).

\section{ROC Analysis}

ROC analysis was applied to calculate the threshold value for the lead time, and a significant difference was detected between attenders and nonattenders. ROC analysis indicated that the AUC was 0.809 (95\%

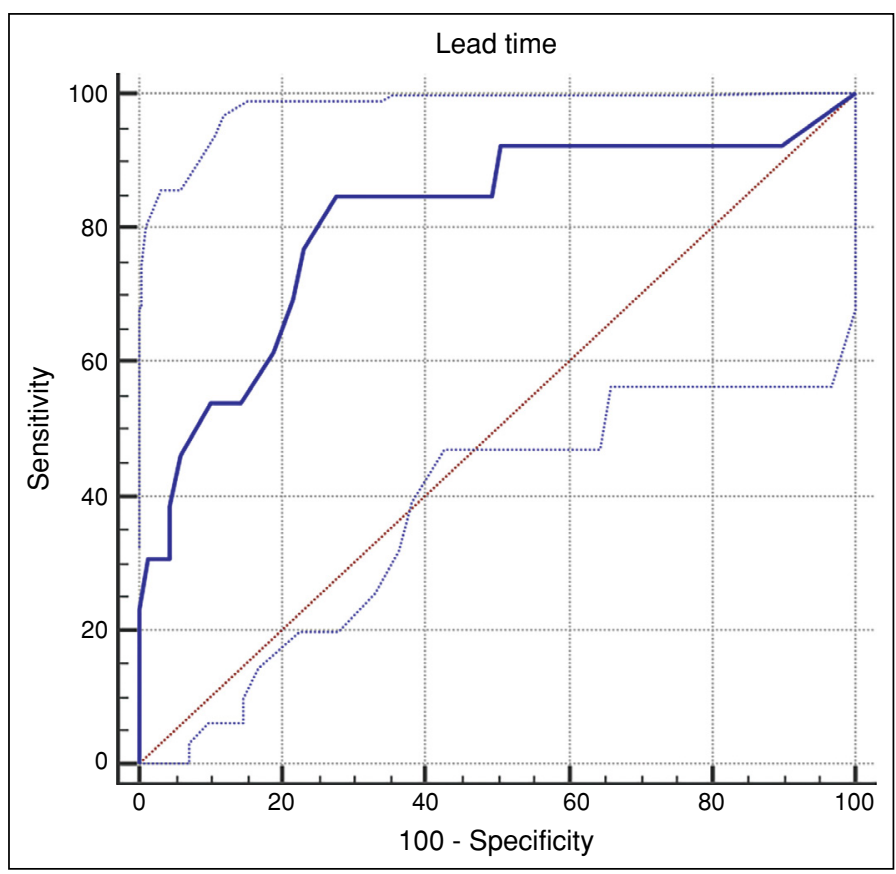

Figure 2. Receiver-operating characteristic curve analysis of the lead time.

CI: 0.708-0.888; p<0.0001) (Fig. 2). The discriminant cut-off value of appointment lead time was 12 days. According to the Youden index, the sensitivity was $85.62 \%$, and the specificity was $72.4 \%$. The accuracy was determined to be 0.74 . The positive likelihood ratio, negative likelihood ratio, positive predictive value, and negative predictive values were $3.07,0.21,36.7$, and 96.2, respectively.

\section{Discussion}

A liver biopsy is the gold standard diagnostic method to evaluate liver pathologies, despite the development of non-invasive techniques. Failure to attend an appointment for a biopsy leads to the inefficient use of health resources and delays in diagnosis and treatment. Targeted intervention that could enhance the effectiveness of hepatology clinics has substantial value. A determination of the reasons for nonattendance at biopsy appointments and the factors that lead to nonattendance could be of great benefit.

Our results showed that $15.9 \%$ of the scheduled patients did not attend scheduled appointments for a liver biopsy. The time interval between making the liver biopsy appointment and the appointment date was associated with nonadherence. Our results indicated that a lead time of 12 days significantly determined nonattenders with a sensitivity of $85 \%$ and specificity of $72 \%$. There was no association between nonattendance and age, gender, travel distance, travel time, the season of the scheduled appointment, or malignancy as a comorbidity.

Reported no-show rates vary by specialty and country. According to a recently published systematic literature review that included different countries and disciplines, the average nonattendance rate was $23 \%{ }^{[8]}$ The no-show rate at gastroenterology endoscopy units has been reported to be $3.6 \%$ to $67 \%,{ }^{[9,10]}$ whereas the corresponding rate at gastroenterology outpatient clinic appointments has been reported to be only $29 \%{ }^{[11]}$ We determined a no-show rate for a liver biopsy appointment of $15.9 \%$, which is slightly lower than the average rate in the exist- 
ing literature. ${ }^{[8]}$ One explanation for this variation is that the previous study populations included different procedures, whereas our study comprised patients who underwent only 1 type of procedure. Appointments for various procedures could easily affect the rate of attendance. ${ }^{\left[{ }^{8-10]}\right.}$ Another reason for discrepancies may be that we included patients who had already scheduled a liver biopsy. Studies that have included patients who were simply referred for analysis could result in a higher no-show rate. ${ }^{[12]}$ Additionally, the no-show rate has been shown to be greater among direct to endoscopy ${ }^{[9]}$ and direct endoscopy referral systems. ${ }^{[10]}$ However, an immediate biopsy cannot be performed because a liver biopsy requires blood work, such as coagulation parameters and platelet count, before the procedure. Given all of the various factors, it is understandable that the rate of not attending appointments varies for different procedures and clinics, and it is important to note that nonadherence can lead to inefficiency at hepatology clinics. High nonattendance rates and the subsequent lost time should be eliminated in order to provide better healthcare delivery.

As the time between the liver biopsy appointment date and performance of the procedure grew, we found that the rate nonattendance increased. Patients who are on long waiting lists may forget their appointments. This result supports the use of appointment reminders by phone ${ }^{[13]}$ or text message, ${ }^{[14]}$ which have been shown to increase attendance rates for endoscopy units appointments. Moreover, patients may think that they do not need the procedure because their complaints subside during the waiting period. Patients may also choose to have their biopsy done at another center rather than wait due to the anxiety related to delayed diagnosis and treatment. Patients with a pre-existing awareness of disease may demonstrate more patience, but those with an acute state of uncertainty may be inclined to seek alternative solutions rather than waiting for the appointment. Parker et al. ${ }^{[15]}$ investigated the extent of distress related to gastrointestinal endoscopy and found that $21 \%$ of the patients on the waiting list had a moderate to high level of anxiety, while the rate among the general population was $13 \%$. Increasing anxiety while waiting for an appointment could lead patients to alternative routes, and may be a reason why patients do not attend appointments.

According to the British guidelines, ${ }^{[16]}$ it is recommended that the time between request for a biopsy and completion should be $<4$ weeks as a quality standard. However, it is noteworthy that this recommendation was based upon expert opinion, rather than a study. Our results suggest a shorter interval of 12 days to ensure better compliance. If future studies with broader study populations confirm our results, the recommendations in the guidelines may be revised. Lead time is a modifiable factor for nonattendance, and it would benefit clinics to enhance the adherence to scheduled liver biopsy appointments.

Although previous studies have found that travel distance and travel time were associated with nonattendance, ${ }^{[17,18]}$ we did not find a similar relationship. Our facility is located at the center of a small city; the furthest settlement is $90 \mathrm{~km}$ from our hospital and the average travel time is 1 hour. Though travel distance can be a significant barrier for patients from rural areas, it was not significant in our study conducted in a small city. We also hypothesized that age, gender, the season of the scheduled appointment, or a malignancy comorbidity might be associated with nonattendance; however, our results did not reveal any association. The reason for this discrepancy with the existing literature could be due to the relatively small size of our study population, which may not reveal minor differences. Further studies with a large population may show an association with these parameters.
Limitations of our study include the retrospective design, which may lead to selection bias. However, our electronic records are very reliable, and we excluded inconsistent data to ensure the best analysis. Secondly, nonadherent patients could have been interviewed. It would have been useful to know the reasons they did not attend appointments, but this is the subject of another study currently in progress.

In conclusion, patient nonattendance to a liver biopsy appointment is a significant barrier to treatment and the efficient operation of hepatology clinics. A liver biopsy appointment scheduled more than 2 weeks in advance was associated with a failure to appear. Future studies with a larger and broader population are needed to more specifically identify factors associated with nonattendance in order to improve the effectiveness of hepatology outpatient clinic performance and patient treatment.

Acknowledgement: The author is grateful for assisting in data extraction to Dr. Burcu Kocyigit (Department of Internal Medicine, Kocaeli University School of Medicine) and valuable support in statistical analysis to Dr. Sibel Balci (Department of Biostatistics, Kocaeli University School of Medicine).

Ethics Committee Approval: The Kocaeli University Clinical Research Ethics Committee granted approval for this study (date: 18.02.2021, number: 2021/79).

Peer-review: Externally peer-reviewed.

Conflict of Interest: The author have no conflict of interest to declare.

Financial Disclosure: The author declared that this study has received no financial support.

\section{References}

1. Poynard T, Ratziu V, Bedossa P. Appropriateness of liver biopsy. Can J Gastroenterol 2000;14(6):543-548. [CrossRef]

2. Cadranel J-F, Rufat P, Degos F. Practices of liver biopsy in France: results of a prospective nationwide survey. Hepatology 2000;32(3):477-481. [CrossRef]

3. McGill DB, Rakela J, Zinsmeister AR, Ott BJ. A 21-year experience with major hemorrhage after percutaneous liver biopsy. Gastroenterology 1990;99(5):1396-1400. [CrossRef]

4. Poynard T, McHutchison J, Manns M, Myers RP, Albrecht J. Biochemical surrogate markers of liver fibrosis and activity in a randomized trial of peginterferon alfa-2b and ribavirin. Hepatology. 2003;38(2):481-492. [CrossRef]

5. Thuluvath PJ, Krok KL. Noninvasive markers of fibrosis for longitudinal assessment of fibrosis in chronic liver disease: are they ready for prime time? Am J Gastroenterol 2006;101(7):1497-1499. [CrossRef]

6. Ziol M, Handra-Luca A, Kettaneh A, Christidis C, Mal F, Kazemi F, et al. Non-invasive assessment of liver fibrosis by measurement of stiffness in patients with chronic hepatitis C. Hepatology 2005;41(1):48-54. [CrossRef]

7. Angel W, Hawkins CM, Wang JM, Hughes DR, Duszak R. Percutaneous hepatic and renal biopsy procedures: an 18-year analysis of changing utilization, specialty roles, and sites of service. J Vasc Interv Radiol 2015;26:680685. [CrossRef]

8. Dantas LF, Fleck JL, Cyrino Oliveira FL, Hamacher S. No-shows in appointment scheduling - a systematic literature review. Health Policy 2018;122(4):412-421. [CrossRef]

9. Wong VK, Zhang HB, Enns R. Factors associated with patient absenteeism for scheduled endoscopy. World J Gastroenterol 2009;15(23):2882-2886.

10. Nash D, Azeez S, Vlahov D, Schori M. Evaluation of an intervention to increase screening colonoscopy in an urban public hospital setting. J Urban Heal 2006;83(2):231-243. [CrossRef]

11. Umar J, Pantangi P, Rawlins S. High no-show rates in the outpatient clinic: analysis of a University Based Gastroenterology (GI) practice: 950. Off J Am Coll Gastroenterol 2016;111:S411. [CrossRef]

12. Naylor K, Ward J, Polite BN. Interventions to improve care related to colorectal cancer among racial and ethnic minorities: a systematic review. J 
Gen Intern Med 2012;27(8):1033-1046. [CrossRef]

13. Childers RE, Laird A, Newman L, Keyashian K. The role of a nurse telephone call to prevent no-shows in endoscopy. Gastrointest Endosc 2016;84(6):1010-1017.e1. [CrossRef]

14. Lam TYT, Hui AJ, Sia F, Wong MY, Lee CCP, Chung KW, et al. Short message service reminders reduce outpatient colonoscopy nonattendance rate: a randomized controlled study. J Gastroenterol Hepatol 2021;36(4):10441050. [CrossRef]

15. Parker J, Kennedy P. Factors predictive of distress in people awaiting a lower gastro-intestinal endoscopy. Psychol Health Med 2010;15(1):26-33. [CrossRef]
16. Neuberger J, Patel J, Caldwell H, Davies S, Hebditch V, Hollywood C, et al. Guidelines on the use of liver biopsy in clinical practice from the British Society of Gastroenterology, the Royal College of Radiologists and the Royal College of Pathology. Gut 2020;69(8):1382-1403. [CrossRef]

17. Lester S, Harris SM. Factors associated with first session nonattendance at a university-based family therapy clinic. Am J Fam Ther 2007;35(4):363-376.

18. Guay MO, Tanzi S, Arregui MT, Chisholm G, del a Cruz M, Bruera E. Characteristics and outcomes of advanced cancer patients who miss outpatient supportive care consult appointments. Support Care Cancer 2014;22(10):2869-2874. [CrossRef] 